\title{
GLOBAL INTEGRABILITY OF THE JACOBIAN OF A COMPOSITE MAPPING
}

\author{
SHUSEN DING AND BING LIU
}

Received 18 September 2005; Accepted 24 October 2005

We first obtain an improved version of the Hölder inequality with Orlicz norms. Then, as an application of the new version of the Hölder inequality, we study the integrability of the Jacobian of a composite mapping. Finally, we prove a norm comparison theorem.

Copyright (c) 2006 S. Ding and B. Liu. This is an open access article distributed under the Creative Commons Attribution License, which permits unrestricted use, distribution, and reproduction in any medium, provided the original work is properly cited.

\section{Introduction}

Carl Gustav Jacob Jacobi (1804-1851), one of the nineteenth century Germany's most accomplished scientists, developed the theory of determinants and transformations into a powerful tool for evaluating multiple integrals and solving differential equations. Since then, the Jacobian (determinant) has played a critical role in multidimensional analysis and related fields, including nonlinear elasticity, weakly differentiable mappings, continuum mechanics, nonlinear PDEs, and calculus of variations. The integrability of Jacobians has become a rather important topic in the study of Jacobians because one of the major applications of Jacobians is to evaluate multiple integrals. Higher integrability properties of the Jacobian first showed up in [2], where Gehring invented reverse Hölder inequalities and used these inequalities to establish the $L^{1+\varepsilon}$-integrability of the Jacobian of a quasiconformal mapping, $\varepsilon>0$. Recently, the integrability of Jacobians of orientation-preserving mappings of Sobolev class $W_{\text {loc }}^{1, n}\left(\Omega, \mathbb{R}^{n}\right)$ has attracted the attention of mathematicians, see [1,3-7], for instance. The purpose of this paper is to study the $L^{p}(\log L)^{\alpha}(\Omega)$-integrability of the Jacobian of a composite mapping.

Let $0<p<\infty$ and $\alpha \geq 0$ be real numbers and let $E$ be any subset of $\mathbb{R}^{n}$. We define the functional on a measurable function $f$ over $E$ by

$$
[f]_{L^{p}(\log L)^{\alpha}(E)}=\left(\int_{E}|f|^{p} \log ^{\alpha}\left(e+\frac{|f|}{\|f\|_{p}}\right) d x\right)^{1 / p}
$$

Hindawi Publishing Corporation Journal of Inequalities and Applications Volume 2006, Article ID 89134, Pages 1-9 DOI 10.1155/JIA/2006/89134 
2 Global integrability of the Jacobian of a composite mapping

where $\|f\|_{p}=\left(\int_{E}|f(x)|^{p} d x\right)^{1 / p}$. In this paper, we always assume that $\Omega$ is a bounded open subset of $\mathbb{R}^{n}, n \geq 2$. We write $L^{p}(\log L)^{\alpha}(\Omega)$ for the space of all measurable functions $f$ on $\Omega$ such that $[f]_{L^{p}(\log L)^{\alpha}(\Omega)}<\infty$. As usual, we simply write $L^{p}(\Omega)=L^{1}(\log L)^{0}(\Omega)$ and $L \log L(\Omega)=L^{1}(\log L)^{1}(\Omega)$, respectively.

A continuously increasing function $\varphi:[0, \infty] \rightarrow[0, \infty]$ with $\varphi(0)=0$ and $\varphi(\infty)=\infty$ is called an Orlicz function. The Orlicz space $L^{\varphi}(\Omega)$ consists of all measurable functions $f$ on $\Omega$ such that

$$
\int_{\Omega} \varphi\left(\frac{|f|}{\lambda}\right) d x<\infty
$$

for some $\lambda=\lambda(f)>0 . L^{\varphi}(\Omega)$ is equipped with the nonlinear Luxemburg functional

$$
\|f\|_{\varphi}=\inf \left\{\lambda>0: \int_{\Omega} \varphi\left(\frac{|f|}{\lambda}\right) d x \leq 1\right\}
$$

A convex Orlicz function $\varphi$ is often called a Young function. If $\varphi$ is a Young function, then $\|\cdot\|_{\varphi}$ defines a norm in $L^{\varphi}(\Omega)$, which is called the Luxemburg norm. For $\varphi(t)=$ $t^{p} \log ^{\alpha}(e+t), 0<p<\infty$ and $\alpha \geq 0$, we have

$$
\|f\|_{L^{p} \log ^{\alpha} L}=\inf \left\{k: \int_{\Omega}|f|^{p} \log ^{\alpha}\left(e+\frac{|f|}{k}\right) d x \leq k^{p}\right\} .
$$

From Theorem 4.2 that will be proved later in this paper, we see that the Luxemburg norm $\|f\|_{\varphi}$ is equivalent to $[f]_{L^{p}(\log L)^{\alpha}(\Omega)}$ defined in (1.1) for any $0<p<\infty$ and $\alpha \geq 0$. Hence, the Orlicz space $L^{\psi}(\Omega)$ with $\psi(t)=t^{p} \log ^{\alpha}(e+t)$ can be denoted by $L^{p}(\log L)^{\alpha}(\Omega)$ and the corresponding norm can also be written as $[f]_{L^{p}(\log L)^{\alpha}(\Omega)}$. The following version of Hölder inequality appears in [3, Proposition 2.2].

Theorem 1.1. Let $1<p, q<\infty, \alpha, \beta>0,1 / p+1 / q=1 / r, \alpha / p+\beta / q=\gamma / r$ and $f \in$ $L^{p}(\log L)^{\alpha}(\Omega), g \in L^{q}(\log L)^{\beta}(\Omega)$. Then $f g \in L^{r}(\log L)^{\gamma}(\Omega)$ and

$$
\|f g\|_{L^{r} \log ^{\gamma} L} \leq C\|f\|_{L^{p} \log ^{\alpha} L}\|g\|_{L^{q} \log ^{\beta} L}
$$

In this paper, we improve the condition $1<p, q<\infty$ into $0<p, q<\infty$ in Theorem 2.1. We enjoy the elementary method used in the proof of Theorem 2.1. Then, using the improved Hölder inequality, we study the $L^{p}(\log L)^{\alpha}(\Omega)$-integrability of the Jacobian of the composition of mappings.

\section{Improved Hölder inequality}

Using Theorem 1.1 and the basic properties of logarithmic functions, we have the following generalized Hölder inequality. 
Theorem 2.1. Let $m, n, \alpha, \beta>0,1 / s=1 / m+1 / n, \alpha / m+\beta / n=\gamma / s$. Assume that $f \in L^{m}$ $(\log L)^{\alpha}(\Omega)$ and $g \in L^{n}(\log L)^{\beta}(\Omega)$. Then, $f g \in L^{s}(\log L)^{\gamma}(\Omega)$ and

$$
\begin{aligned}
& \left(\int_{\Omega}|f g|^{s} \log ^{\gamma}\left(e+\frac{|f g|}{\|f g\|_{s}}\right) d x\right)^{1 / s} \\
& \quad \leq C\left(\int_{\Omega}|f|^{m} \log ^{\alpha}\left(e+\frac{|f|}{\|f\|_{m}}\right) d x\right)^{1 / m}\left(\int_{\Omega}|g|^{n} \log ^{\beta}\left(e+\frac{|g|}{\|g\|_{n}}\right) d x\right)^{1 / n},
\end{aligned}
$$

where $C$ is a positive constant.

Note that (2.1) can be written as

$$
[f g]_{L^{s}(\log L)^{\gamma}(\Omega)} \leq C[f]_{L^{m}(\log L)^{\alpha}(\Omega)}[g]_{L^{n}(\log L)^{\beta}(\Omega)}
$$

Proof. Using the elementary inequality $\log \left(e+x^{a}\right) \leq \log (e+x)^{a+1}$ for $a>0, x>0$, we have

$$
\begin{gathered}
\log \left(e+\frac{\left(|f|^{s}|g|^{s}\right)^{1 / s}}{\left\||f|^{s}|g|^{s}\right\|_{1}^{1 / s}}\right) \leq \log \left(e+\frac{|f|^{s}|g|^{s}}{\left\||f|^{s}|g|^{s}\right\|_{1}}\right)^{1 / s+1}=\left(\frac{1}{s}+1\right) \log \left(e+\frac{|f|^{s}|g|^{s}}{\left\||f|^{s}|g|^{s}\right\|_{1}}\right) \\
\log \left(e+\left(\frac{|f|}{\|f\|_{m}}\right)^{s}\right) \leq \log \left(e+\frac{|f|}{\|f\|_{m}}\right)^{s+1} \leq(s+1) \log \left(e+\frac{|f|}{\|f\|_{m}}\right), \\
\log \left(e+\left(\frac{|g|}{\|g\|_{n}}\right)^{s}\right) \leq \log \left(e+\frac{|g|}{\|g\|_{n}}\right)^{s+1} \leq(s+1) \log \left(e+\frac{|g|}{\|g\|_{n}}\right) .
\end{gathered}
$$

From Hölder inequality (1.5) with $1=1 / m / s+1 / n / s$ (note that $m / s>1, n / s>1$ since $1 / s=1 / m+1 / n)$ and $(2.3)$, we have

$$
\begin{aligned}
& \int_{\Omega}|f g|^{s} \log ^{\gamma}\left(e+\frac{|f g|}{\|f g\|_{s}}\right) d x \\
& =\int_{\Omega}\left(|f|^{s}|g|^{s}\right) \log ^{\gamma}\left(e+\frac{\|\left.\left. f\right|^{s}|g|^{s}\right|^{1 / s}}{\|\left.|f|^{s}|g|^{s}\right|_{1} ^{1 / s}}\right) d x \\
& \leq C_{1} \int_{\Omega}\left(|f|^{s}|g|^{s}\right) \log ^{\gamma}\left(e+\frac{|f|^{s}|g|^{s}}{\|\left.|f|^{s}|g|^{s}\right|_{1}}\right) d x \\
& \leq C_{2}\left(\int_{\Omega}\left(|f|^{s}\right)^{m / s} \log ^{\alpha}\left(e+\frac{|f|^{s}}{\|\left.|f|^{s}\right|_{m / s}}\right) d x\right)^{s / m}\left(\int_{\Omega}\left(|g|^{s}\right)^{n / s} \log ^{\beta}\left(e+\frac{|g|^{s}}{\|\left.|g|^{s}\right|_{n / s}}\right) d x\right)^{s / n} \\
& =C_{2}\left(\int_{\Omega}|f|^{m} \log ^{\alpha}\left(e+\left(\frac{|f|}{\|f\|_{m}}\right)\right) d x\right)^{s / m}\left(\int_{\Omega}|g|^{n} \log ^{\beta}\left(e+\left(\frac{|g|}{\|g\|_{n}}\right)\right) d x\right)^{s / n} \\
& \leq C_{3}\left(\int_{\Omega}|f|^{m} \log ^{\alpha}\left(e+\frac{|f|}{\|f\|_{m}}\right) d x\right)^{s / m}\left(\int_{\Omega}|g|^{n} \log ^{\beta}\left(e+\frac{|g|}{\|g\|_{n}}\right) d x\right)^{s / n} \text {. }
\end{aligned}
$$


4 Global integrability of the Jacobian of a composite mapping

Hence, we conclude that

$$
\begin{aligned}
& \left(\int_{\Omega}|f g|^{s} \log ^{\gamma}\left(e+\frac{|f g|}{\|f g\|_{s}}\right) d x\right)^{1 / s} \\
& \quad \leq C_{4}\left(\int_{\Omega}|f|^{m} \log ^{\alpha}\left(e+\frac{|f|}{\|f\|_{m}}\right) d x\right)^{1 / m}\left(\int_{\Omega}|g|^{n} \log ^{\beta}\left(e+\frac{|g|}{\|g\|_{n}}\right) d x\right)^{1 / n} .
\end{aligned}
$$

The proof of Theorem 2.1 has been completed.

From Theorem 2.1, we have the following general result immediately.

Corrollary 2.2. Let $p_{i}>0, \alpha_{i}>0$ for $i=1,2, \ldots, k, 1 / p_{1}+1 / p_{2}+\cdots+1 / p_{k}=1 / p$, and $\alpha_{1} / p_{1}+\alpha_{2} / p_{2}+\cdots+\alpha_{k} / p_{k}=\alpha / p$. Assume that $f_{i} \in L^{p_{i}}(\log L)^{\alpha_{i}}(\Omega)$ for $i=1,2, \ldots, k$. Then $f_{1} f_{2} \cdots f_{k} \in L^{p}(\log L)^{\alpha}(\Omega)$ and

$$
\left[f_{1} f_{2} \cdots f_{k}\right]_{L^{p}(\log L)^{\alpha}(\Omega)} \leq C\left[f_{1}\right]_{L^{p_{1}}(\log L)^{\alpha_{1}}(\Omega)}\left[f_{2}\right]_{L^{p_{2}(\log L)^{\alpha_{2}}(\Omega)}} \cdots\left[f_{k}\right]_{L^{p_{k}(\log L)^{\alpha_{k}}(\Omega)}},
$$

where $C$ is a positive constant and the norms $\left[f_{1} f_{2} \cdots f_{k}\right]_{L^{p}(\log L)^{\alpha}(\Omega)}$ and $\left[f_{i}\right]_{L^{p_{i}}(\log L)^{\alpha_{i}}(\Omega)}$, $i=1,2, \ldots, k$, are defined in (1.1).

\section{Integrability of Jacobians of composite mappings}

In this section, we explore applications of the new version of the Hölder inequality established in the last section. Specifically, we study the integrability of the Jacobian of the composition of mappings $f: \Omega \rightarrow \mathbb{R}^{n}, f=\left(f^{1}\left(u_{1}, u_{2}, \ldots, u_{n}\right), f^{2}\left(u_{1}, u_{2}, \ldots, u_{n}\right), \ldots, f^{n}\left(u_{1}, u_{2}\right.\right.$, $\left.\left.\ldots, u_{n}\right)\right)$ of Sobolev class $W_{\text {loc }}^{1, p}\left(\Omega, \mathbb{R}^{n}\right)$, where $u_{i}=u_{i}\left(x_{1}, x_{2}, \ldots, x_{n}\right), i=1,2, \ldots, n$, are functions of $x=\left(x_{1}, x_{2}, \ldots, x_{n}\right) \in \Omega$ with continuous partial derivatives $\partial u_{i} / \partial x_{j}, j=1,2, \ldots, n$. Assume that the distributional differential $D f(u)=\left[\partial f^{i} / \partial u_{j}\right]$ and $D u(x)=\left[\partial u_{i} / \partial x_{j}\right]$ are locally integrable functions with values in the space $\mathrm{GL}(n)$ of all $n \times n$-matrices. As usual, we write

$$
\begin{aligned}
& J(x, f)=\operatorname{det} D f(u(x))=\frac{\partial\left(f^{1} \cdots f^{n}\right)}{\partial\left(x_{1} \cdots x_{n}\right)}, \\
& J(u, f)=\operatorname{det} D f(u)=\frac{\partial\left(f^{1} \cdots f^{n}\right)}{\partial\left(u_{1} \cdots u_{n}\right)}, \\
& J(x, u)=\operatorname{det} D u(x)=\frac{\partial\left(u_{1} \cdots u_{n}\right)}{\partial\left(x_{1} \cdots x_{n}\right)},
\end{aligned}
$$

respectively. Using Theorem 2.1, we have the following integrability theorem for the Jacobian of the composition of mappings.

Theorem 3.1. Let $s, t, \beta, \gamma>0$, with $1 / p=1 / s+1 / t$ and $\beta / s+\gamma / t=\alpha / p$. Assume that $J(x, f), J(u, f)$, and $J(x, u)$ are Jacobians defined in (3.1), (3.2), and (3.3), respectively. If 
$J(u(x), f) \in L^{s}(\log L)^{\beta}(\Omega)$ and $J(x, u) \in L^{t}(\log L)^{\gamma}(\Omega)$, then $J(x, f) \in L^{p}(\log L)^{\alpha}(\Omega)$ and

$$
\begin{aligned}
\left(\int_{\Omega}|J(x, f)|^{p} \log ^{\alpha}\left(e+\frac{|J(x, f)|}{\|J(x, f)\|_{p}}\right) d x\right)^{1 / p} \\
\leq C\left(\int_{\Omega}|J(u, f)|^{s} \log ^{\beta}\left(e+\frac{|J(u, f)|}{\|J(u, f)\|_{s}}\right) d x\right)^{1 / s} \\
\quad \times\left(\int_{\Omega}|J(x, u)|^{t} \log ^{\gamma}\left(e+\frac{|J(x, u)|}{\|J(x, u)\|_{t}}\right) d x\right)^{1 / t},
\end{aligned}
$$

where $C$ is a positive constant.

Proof. Note that the Jacobian of the composition of $f$ and $u$ can be expressed as

$$
J(x, f)=\frac{\partial\left(f^{1} \cdots f^{n}\right)}{\partial\left(x_{1} \cdots x_{n}\right)}=\frac{\partial\left(f^{1} \cdots f^{n}\right)}{\partial\left(u_{1} \cdots u_{n}\right)} \cdot \frac{\partial\left(u_{1} \cdots u_{n}\right)}{\partial\left(x_{1} \cdots x_{n}\right)}=J(u, f) \cdot J(x, u) .
$$

Applying Theorem 2.1 and (3.5) yields

$$
\begin{aligned}
\left(\int_{\Omega}|J(x, f)|^{p} \log ^{\alpha}\left(e+\frac{|J(x, f)|}{\left.|| J(x, f)\right|_{p}}\right) d x\right)^{1 / p} \\
=\left(\int_{\Omega}|J(u, f) \cdot J(x, u)|^{p} \log ^{\alpha}\left(e+\frac{|J(u, f) \cdot J(x, u)|}{\left.|| J(u, f) \cdot J(x, u)\right|_{p}}\right) d x\right)^{1 / p} \\
\leq C\left(\int_{\Omega}|J(u, f)|^{s} \log ^{\beta}\left(e+\frac{|J(u, f)|}{|| J(u, f) \|_{s}}\right) d x\right)^{1 / s} \\
\quad \times\left(\int_{\Omega}|J(x, u)|^{t} \log ^{\gamma}\left(e+\frac{|J(x, u)|}{\|J(x, u)\|_{t}}\right) d x\right)^{1 / t}<\infty
\end{aligned}
$$

since $J(u(x), f) \in L^{s}(\log L)^{\beta}(\Omega)$ and $J(x, u) \in L^{t}(\log L)^{\gamma}(\Omega)$. Thus, $J(x, f) \in L^{p}(\log L)^{\alpha}(\Omega)$ from (3.6). The proof of Theorem 3.1 has been completed.

Applying the Hölder inequality with $L^{p}$-norms

$$
\|f g\|_{s, E} \leq\|f\|_{\alpha, E} \cdot\|g\|_{\beta, E}
$$

where $0<\alpha, \beta<\infty, s^{-1}=\alpha^{-1}+\beta^{-1}$, and $f$ and $g$ are any measurable functions on a measurable set $E \subset \mathbb{R}^{n}$, we have the following $L^{p}$-integrability theorem for the Jacobian of a composite mapping.

Theorem 3.2. Let $J(x, f), J(u, f)$, and $J(x, u)$ be the Jacobians defined in (3.1), (3.2), and (3.3), respectively. If $J(u(x), f) \in L^{s}(\Omega)$ and $J(x, u) \in L^{t}(\Omega), s, t>0$, then $J(x, f) \in L^{p}(\Omega)$ and

$$
\|J(x, f)\|_{L^{p}(\Omega)} \leq C\|J(u(x), f)\|_{L^{s}(\Omega)}\|J(x, u)\|_{L^{t}(\Omega)},
$$


6 Global integrability of the Jacobian of a composite mapping

where $C$ is a positive constant and the integrability exponent $p$ of $J(x, f)$ determined by $1 / p=1 / s+1 / t$ is the best possible.

The following example shows that the integrability exponent $p$ of $J(x, f)$ cannot be improved anymore.

Example 3.3. We consider the mappings

$$
\begin{gathered}
f(x, y)=\left(f^{1}, f^{2}\right)=\left(\frac{x}{\left(x^{2}+y^{2}\right)^{\sigma}}, \frac{y}{\left(x^{2}+y^{2}\right)^{\sigma}}\right), \quad(x, y) \in D=\left\{(x, y): 0<x^{2}+y^{2} \leq \rho^{2}\right\}, \\
x=r^{-k} \cos \theta, \quad y=r^{-k} \sin \theta, \quad(r, \theta) \in \Omega=\{(r, \theta): 0<r<\rho, 0<\theta \leq 2 \pi\},
\end{gathered}
$$

where $\sigma$ and $\rho$ are positive constants. After a simple calculation, we obtain the following Jacobians:

$$
\begin{gathered}
J_{1}=\frac{\partial\left(f^{1}, f^{2}\right)}{\partial(r, \theta)}=\frac{k(2 \sigma-1)}{r^{4 \sigma+2 k+1}}, \quad J_{2}=\frac{\partial\left(f^{1}, f^{2}\right)}{\partial(x, y)}=\frac{1-2 \sigma}{r^{4 \sigma}}, \\
J_{3}=\frac{\partial(x, y)}{\partial(r, \theta)}=\frac{-k}{r^{2 k+1}}, \quad 0<r<\rho .
\end{gathered}
$$

It is easy to see that $J_{1} \in L^{1 /(4 \sigma+2 k+1)}(\Omega)$ but $J_{1} \notin L^{p}(\Omega)$ for any $p>1 /(4 \sigma+2 k+1)$. Similarly, $J_{2} \in L^{1 / 4 \sigma}(\Omega)$ but $J_{2} \notin L^{s}(\Omega)$ for any $s>1 / 4 \sigma$ and $J_{3} \in L^{1 /(2 k+1)}(\Omega)$ but $J_{3} \notin L^{t}(\Omega)$ for any $t>1 /(2 k+1)$. Here, the integrability exponent $p=1 /(4 \sigma+2 k+1)$ of $\partial\left(f^{1}, f^{2}\right) / \partial$ $(r, \theta)$ is determined by

$$
\frac{1}{p}=(4 \sigma+2 k+1)=\frac{1}{s}+\frac{1}{t}
$$

where $s=1 / 4 \sigma$ and $t=1 /(2 k+1)$ are the integrability exponents of Jacobians $\partial\left(f^{1}, f^{2}\right) / \partial(x, y)$ and $\partial(x, y) / \partial(r, \theta)$, respectively.

The above example shows that, in Theorem 3.2, the integrability exponent $p$ of $J(x, f)$ that is determined by $1 / p=1 / s+1 / t$ is the best possible, where $s$ is the integrability exponent of $J(u(x), f)$ and $t$ is the integrability exponent of $J(x, u)$.

Example 3.4. Let $J_{1}=\partial\left(f^{1}, f^{2}\right) / \partial(r, \theta), J_{2}=\partial\left(f^{1}, f^{2}\right) / \partial(x, y)$, and $J_{3}=\partial(x, y) / \partial(r, \theta)$ be the Jacobians obtained in Example 3.3. For any $\varepsilon>0$, there exists a constant $C_{1}>0$ such that

$$
\left|J_{1}\right|^{p} \log \left(e+\frac{\left|J_{1}\right|}{|| J_{1} \|_{p}}\right) \leq C_{1}\left|J_{1}\right|^{p+\varepsilon /(4 \sigma+2 k+1)}
$$


Using (3.10) and (3.12), we have

$$
\begin{aligned}
\int_{\Omega}\left|J_{1}\right|{ }^{p} \log \left(e+\frac{\left|J_{1}\right|}{\left\|J_{1}\right\|_{p}}\right) d r d \theta \\
\quad=2 \pi \int_{0}^{\rho}\left|J_{1}\right|^{p} \log \left(e+\frac{\left|J_{1}\right|}{\left\|J_{1}\right\|_{p}}\right) d r \\
\quad=C_{2} \int_{0}^{\rho}\left(\frac{1}{r^{4 \sigma+2 k+1}}\right)^{p} \log \left(e+\frac{\left|(2 \sigma-1) k / r^{4 \sigma+2 k+1}\right|}{\left\|(2 \sigma-1) k / r^{4 \sigma+2 k+1}\right\|_{p}}\right) d r \\
\quad \leq C_{3} \int_{0}^{\rho} r^{-(4 \sigma+2 k+1) p}\left(r^{-(4 \sigma+2 k+1)}\right)^{\varepsilon / 4 \sigma+2 k+1} d r \leq C_{4} \int_{0}^{\rho} r^{-(4 \sigma+2 k+1) p-\varepsilon} d r=C_{5}<\infty
\end{aligned}
$$

for any $p$ satisfying $0<p \leq 1 /(4 \sigma+2 k+1)-\varepsilon /(4 \sigma+2 k+1)$. Since $\varepsilon>0$ is arbitrary, we know that $J_{1} \in L^{p} \log L(\Omega)$ for any $p$ with $0<p<1 /(4 \sigma+2 k+1)$. Similarly, we have $J_{2} \in$ $L^{s} \log L(\Omega)$ for any $s$ with $0<s<1 / 4 \sigma$ and $J_{3} \in L^{t} \log L(\Omega)$ for any $t$ with $0<t<1 /(2 k+1)$. This example shows that the integrability exponent $p$ of $\partial\left(f^{1}, f^{2}\right) / \partial(r, \theta)$ that is determined by $1 / p=1 / s+1 / t$ is the best possible when $\alpha=\beta=\gamma=1$ in Theorem 3.1.

\section{The norm comparison theorem}

In this section, we discuss the relationship between norms $\|f\|_{L^{p} \log ^{\alpha} L^{2}}$ and $[f]_{L^{p}(\log L)^{\alpha}(\Omega)}$, which will provide a different way to prove Theorems 2.1 and 3.1. First, we recall the following more general inequality appearing in [3, Theorem A.1].

Theorem 4.1. Suppose that $A, B, C:[0, \infty) \rightarrow[0, \infty)$ are continuous, monotone increasing functions for which there exist positive constants $c$ and $d$ such that

(i) $B^{-1}(t) C^{-1}(t) \leq c A^{-1}(t)$ for all $t>0$,

(ii) $A(t / d) \leq 1 / 2 A(t)$ for all $t>0$.

Suppose that $G$ is an open subset of $\mathbb{R}^{n}$, for $f \in L_{B}(G)$ and $g \in L_{C}(G)$. Then $f g \in L_{A}(G)$ and

$$
\|f g\|_{A} \leq c d\|f\|_{B}\|G\|_{C} .
$$

In [6], Iwaniec and Verde prove that the norm $\|f\|_{L^{p} \log ^{\alpha} L}$ is equivalent to the norm $[f]_{L^{p}(\log L)^{\alpha}(\Omega)}$ for $1<p<\infty$. Similar to the proof of [6, Lemma 8.6], we have the relationship between the norm $\|f\|_{L^{p} \log ^{\alpha} L}$ and the norm $[f]_{L^{p}(\log L)^{\alpha}(\Omega)}$.

Theorem 4.2. For each $f \in L^{p}(\log L)^{\alpha}(\Omega), 0<p<\infty$ and $\alpha \geq 0$,

$$
\|f\|_{p} \leq\|f\|_{L^{p} \log ^{\alpha} L} \leq[f]_{L^{p}(\log L)^{\alpha}(\Omega)} \leq C\|f\|_{L^{p} \log ^{\alpha} L}
$$

where $C=2^{\alpha / p}\left(1+(\alpha / e p)^{\alpha}\right)^{1 / p}$ is a constant independent of $f$. 
8 Global integrability of the Jacobian of a composite mapping

Proof. Let $K=\|f\|_{L^{p} \log ^{\alpha} L}$. Then, by the definition of the Luxemburg norm, we have

$$
K=\left(\int_{\Omega}|f|^{p} \log ^{\alpha}\left(e+\frac{|f|}{K}\right) d x\right)^{1 / p}
$$

It is clear that $K \geq\|f\|_{p}$ and

$$
K \leq\left(\int_{\Omega}|f|^{p} \log ^{\alpha}\left(e+\frac{|f|}{\|f\|_{p}}\right) d x\right)^{1 / p}=[f]_{L^{p}(\log L)^{\alpha}(\Omega)},
$$

that is,

$$
\|f\|_{L^{p} \log ^{\alpha} L} \leq[f]_{L^{p}(\log L)^{\alpha}(\Omega)} .
$$

On the other hand, using $K \geq\|f\|_{p}$ and the elementary inequality $|a+b|^{s} \leq 2^{s}\left(|a|^{s}+\right.$ $\left.|b|^{s}\right), s \geq 0$, we obtain that

$$
\begin{aligned}
\int_{\Omega}|f|^{p} \log ^{\alpha}\left(e+\frac{|f|}{\|f\|_{p}}\right) d x & =\int_{\Omega}|f|^{p} \log ^{\alpha}\left(e+\frac{|f|}{K} \cdot \frac{K}{\|f\|_{p}}\right) d x \\
& \leq \int_{\Omega}|f|^{p}\left(\log \left(e+\frac{|f|}{K}\right)+\log \left(\frac{K}{\|f\|_{p}}\right)\right)^{\alpha} d x \\
& \leq 2^{\alpha} \int_{\Omega}|f|^{p} \log ^{\alpha}\left(e+\frac{|f|}{K}\right)+2^{\alpha} \int_{\Omega}|f|^{p} \log ^{\alpha}\left(\frac{K}{\|f\|_{p}}\right) d x \\
& =2^{\alpha} K^{p}+2^{\alpha}\|f\|_{p}^{p} \log ^{\alpha}\left(\frac{K}{\|f\|_{p}}\right) .
\end{aligned}
$$

Note that the function $h(t)=t^{p} \log ^{\alpha}(K / t), 0<t \leq K$, has its maximum value $(\alpha / e p)^{\alpha} K^{p}$ at $t=K / e^{\alpha / p}$. Then

$$
\|f\|_{p}^{p} \log ^{\alpha}\left(\frac{K}{\|f\|_{p}}\right) \leq\left(\frac{\alpha}{e p}\right)^{\alpha} K^{p} .
$$

Combining (4.6) and (4.7) gives

$$
\int_{\Omega}|f|^{p} \log ^{\alpha}\left(e+\frac{|f|}{\|f\|_{p}}\right) d x \leq 2^{\alpha}\left(1+\left(\frac{\alpha}{e p}\right)^{\alpha}\right) K^{p},
$$

which is equivalent to

$$
[f]_{L^{p}(\log L)^{\alpha}(\Omega)} \leq C\|f\|_{L^{p} \log ^{\alpha} L},
$$

where $C=2^{\alpha / p}\left(1+(\alpha / e p)^{\alpha}\right)^{1 / p}$. The proof of Theorem 4.2 has been completed.

It is easy to see that Theorem 4.2 indicates that, for any $0<p<\infty$ and $\alpha \geq 0$, the Luxemburg norm $\|f\|_{L^{p} \log ^{\alpha} L}$ is equivalent to the norm $[f]_{L^{p}(\log L)^{\alpha}(\Omega)}$ defined in (1.1). Hence, we can also prove Theorems 2.1 and 3.1 using Theorem 4.1 with suitable choices of functions $A(t), B(t)$, and $C(t)$. 


\section{References}

[1] H. Brezis, N. Fusco, and C. Sbordone, Integrability for the Jacobian of orientation preserving mappings, Journal of Functional Analysis 115 (1993), no. 2, 425-431.

[2] F. W. Gehring, The $L^{p}$-integrability of the partial derivatives of a quasiconformal mapping, Acta Mathematica 130 (1973), 265-277.

[3] J. Hogan, C. Li, A. McIntosh, and K. Zhang, Global higher integrability of Jacobians on bounded domains, Annales de l'Institut Henri Poincaré. Analyse Non Linéaire 17 (2000), no. 2, 193-217.

[4] T. Iwaniec and C. Sbordone, On the integrability of the Jacobian under minimal hypotheses, Archive for Rational Mechanics and Analysis 119 (1992), no. 2, 129-143.

[5] __ Weak minima of variational integrals, Journal für die reine und angewandte Mathematik 454 (1994), 143-161.

[6] T. Iwaniec and A. Verde, On the operator $\mathscr{L}(f)=f \log |f|$, Journal of Functional Analysis 169 (1999), no. 2, 391-420.

[7] S. Müller, Higher integrability of determinants and weak convergence in $L^{1}$, Journal für die reine und angewandte Mathematik 412 (1990), 20-34.

Shusen Ding: Department of Mathematics, Seattle University, Seattle, WA 98122, USA

E-mail address: sding@seattleu.edu

Bing Liu: Department of Mathematical Sciences, Saginaw Valley State University, University Center, MI 48710, USA

E-mail address: bliu@svsu.edu 